\title{
Impacto de la crisis petrolera y la caída en la demanda hotelera en la ciudad del Coca
}

DOI: https://doi.org/10.33262/ap.v3i4.117

(c) (i) (5) (2)

Impact of the oil crisis and the fall in hotel demand in the city of Coca

Carlos Andrés Vinueza Chávez. ${ }^{1}$, Rubén Aucancela Chimbolema. ${ }^{2}$ \& Mayra Inés Pinta Rodríguez. $^{3}$

\begin{abstract}
Introduction. The research analyzes the data that allow verifying the impact that the development of the oil sector had on the hotel activity of Coca in the Amazonian north of Ecuador during the period 2010 to 2019. The hotels were characterized for which interviews were applied that allow understanding the reality regarding the perception of the incidence of the oil crisis in its management. In addition, data related to variations in the price of a barrel of crude oil, as well as its production and export are detailed, which are contrasted with the economic evolution of the hotel activity. Methodology. The investigation is qualitative quantitative. The analysis of the primary information collected through interviews is qualitative in which: "the collection of data without numerical measurement is used to describe or refine research questions in the interpretation process" Ander-Egg (1982), and the analysis of the secondary information obtained from the bulletins of the Central Bank of Ecuador is quantitative, to determine the intensity of the correlation of the variables under study. The research was based on an inductive process, to obtain perspectives, opinions and points of view from those involved. Results. Pearson's correlation coefficient has a value of 0.810 , which shows a high positive linear correlation between oil exports and hotel demand in the oil segment in the city of Coca. The higher the oil revenues, the hotel demand in this segment increases and when an oil

\footnotetext{
1 Universidad Estatal Amazónica, Facultad de Ciencias de la Vida. Puyo, Ecuador. ca.vinuezach@uea.edu.ec, https://orcid.org/0000-0002-4174-9208

2 Universidad Estatal Amazónica, Facultad de Ciencias de la Vida. Puyo, Ecuador. raucancelach@uea.edu.ec, https://orcid.org/0000-0002-7649-978X

${ }^{3}$ Universidad Estatal Amazónica, Facultad de Ciencias de la Vida. Puyo, Ecuador. mpinta@uea.edu.ec, https://orcid.org/0000-0002-8285-5381
} 
crisis occurs, the hotel demand drops. The statistical significance measured at two tails was 0.004 , which indicates a highly significant dependency relationship between oil revenues and hotel demand in the oil segment. The hotel demand based on tourists from the Yasuní National Park was not significant.

Keywords. - Incidence; oil; development; management; hospitality.

\section{Resumen}

Introducción. La investigación analiza los datos que permiten constatar la incidencia que tuvo el desarrollo del sector petrolero en la actividad hotelera del Coca en el norte amazónico del Ecuador durante el periodo 2010 al 2019. Se caracterizaron los hoteles para lo cual se aplicaron entrevistas que permiten comprender la realidad respecto a la percepción de la incidencia de la crisis petrolera en su gestión. Además, se detallan datos relacionados a las variaciones del precio por barril de crudo, así como de su producción y exportación que se contrastan con la evolución económica de la actividad hotelera. Metodología. La investigación es de tipo cualicuantitavo. El análisis de la información primaria recabada por medio de entrevistas es cualitativo en la que: "la recolección de datos sin medición numérica se usa para describir o afinar preguntas de investigación en el proceso de interpretación” Ander-Egg (1982), y el análisis de la información secundaria obtenida de los boletines del Banco Central del Ecuador es de tipo cuantitativa, para determinar la intensidad de la correlación de las variables en estudio. La investigación se fundamentó en un proceso inductivo, para obtener perspectivas, opiniones y puntos de vista de los involucrados. Resultados. El coeficiente de correlación de Pearson tiene un valor de 0.810 que evidencia una alta correlación lineal positiva entre las exportaciones de petróleo y la demanda hotelera del segmento petrolero en la ciudad del Coca. Mientras mayores son los ingresos petroleros, la demanda hotelera de este segmento se incrementa y cuando ocurre una crisis petrolera, baja la demanda hotelera. La significancia estadística medida a dos colas fue de 0.004 que indica una relación de dependencia altamente significativa entre los ingresos petroleros y la demanda hotelera del segmento petrolero. La demanda hotelera en función de turistas del Parque Nacional Yasuní no fue significativa.

Palabras Claves. - Incidencia; petróleo; desarrollo; gestión; hotelería.

\section{Introducción}

El cantón Francisco de Orellana se encuentra ubicado al nororiente de la Región Amazónica Ecuatoriana (RAE) 5 y forma parte de la Zona de Planificación 2 conformada por las provincias de Pichincha, Napo y Orellana, provincia a la cual pertenece. El área cantonal ocupa una superficie total de $7.047 \mathrm{~km} 2$ (704.755 ha), en un rango altitudinal que va desde los 100 a los 720 m.s.n.m. Con relación a Quito, capital de Ecuador, la cabecera cantonal Puerto Francisco de Orellana (Coca) se encuentra a una distancia aproximada de 300 km (Alcaldía de Francisco de Orellana, 2019). 
Según Sancho (2019), en el libro de introducción al turismo "Existe un amplio debate académico sobre qué es el turismo, qué elementos lo componen y quién debe ser considerado turista, lo que ha generado multitud de definiciones, cada una subrayando aspectos distintos de la misma actividad". En este sentido, cabe afirmar que no existe definición correcta o incorrecta, ya que todas contribuyen a profundizar el entendimiento del turismo.

La extracción del petróleo en Orellana inicia en la década de los 70 cuando se encontraron grandes yacimientos con políticas de extracción leves, esto permitió que las compañías motivadas por el favorable ambiente para su actividad se multipliquen y se desarrollen generando grandes excedentes económicos y beneficios personales de su actividad con un costo ambiental para el país muy alto.

La economía de la ciudad del Coca, se destaca por ser una importante productora de petróleo, realizando operaciones en el área de explotación de hidrocarburos más importante de Ecuador. La baja del precio de petróleo a nivel internacional y la disminución de la producción, mantienen afectada a la economía local y particularmente se manifiesta con la baja ocupación de la zona hotelera.

Casallas (2014), menciona que "La llegada de estas grandes compañías petroleras estimula la demanda agregada de bienes y servicios, tanto estas empresas como sus empleados que perciben en promedio unos niveles de salarios medios y altos, necesitan vivienda, transporte, alimentación, educación y salud".

Entonces la actividad turística encargada del hospedaje, la alimentación y actividades de distracción se concentró en los recursos naturales y culturales de la zona para generar una propuesta con diferentes hoteles, restaurantes y agencias de viajes con una variedad de servicios.

Según Visión 360 (2021):

De pronto la recesión llegó y generó una serie de despidos en las compañías de petróleo, algunas decidieron empacar y buscar mejores oportunidades, al igual que muchas personas que retornaron a sus ciudades de origen, mientras que los establecimientos turísticos que se dedicaron al segmento corporativo petrolero se quedaron de a poco vacíos hasta caer a finales del 2015 en la quiebra.

Los establecimientos turísticos no previnieron este escenario por lo que su gestión no fue eficiente, y tomaron medidas desesperadas como por ejemplo bajar en un $20 \%$ el precio de sus habitaciones, generar promociones para incentivar a los turistas y por último poner en venta sus negocios.

El objetivo de esta investigación fue: Analizar la incidencia del desarrollo del sector petrolero en la actividad turística de la ciudad del Coca durante el período 2010-2019, partiendo de la caracterización de los establecimientos hoteleros y el desarrollo petrolero 
en Francisco de Orellana y medir el grado de correlación del impacto entre las dos variables.

Goldner y Ritchie (2012), indican que "La teoría económica del turismo muestra que el producto turístico es en efecto un producto compuesto, es decir una mezcla de productos y servicios que se ofertan en los destinos, tales como, las atracciones, hospedajes, transportes y restaurantes".

Para Page (2009), "Sin la existencia de los servicios soportes el producto turístico no puede estructurarse para su consumo", así mismo Xu (2010), considera que "El hospedaje llega a ser el servicio soporte más importante, al servir de apoyo en la estadía de los turistas en cada destino, y el tiempo de estancia de los turistas depende en gran medida del hospedaje".

Según Tafur et al. (2018), "Resulta fundamental que se doten de los instrumentos de Sistemas de información/Tecnologías de información más idóneos que sirva de apoyo a directivos y empleados para mejorar la gestión de la actividad de negocio y la calidad del servicio al cliente".

De hecho, según Guzmán (2004), "La economía turística en esta región se sostiene en la industria petrolera, actualmente en crisis, debido a las nuevas reformas energéticas". El sistema propuesto por Gutiérrez (2013), "Muestra la existencia de dos grupos de factores: endógenos y exógenos, en el núcleo se encuentra el destino turístico, que es el territorio que manifiesta y sobre el cual se manifiestan los demás factores". En este sentido Valls (2004), coincide en que "Este espacio territorial del destino turístico tiene la posibilidad de gestionar su desarrollo en conjunto".

Como factores endógenos en el destino se comprende una relación entre la demanda (turistas y excursionistas que visitan el destino y lo reconocen como tal), y la oferta (atractivos, actividades turísticas, servicios y productos turísticos, la infraestructura y los servicios básicos de apoyo).

Según Caro et al. (2015):

Las potencialidades alcanzadas, han motivado y exigido que el sustento e innovación tecnológica en este sector siga creciendo de manera acelerada, desde los primeros sistemas de información para las reservas de vuelos, a partir de los cuales se generaron los modernos GDS (Sistemas Globales de Distribución), hasta los actuales sistemas de información que copan el ámbito de la gestión.

Para la investigación, la definición de turismo que se adopta es la planteada por Martín (2003):

El conjunto de fenómenos y relaciones económicas, psico-sociológicas y medioambientales que se generan entre las entidades vinculadas a los viajes desde el lugar emisor, las entidades proveedoras de servicios y productos en el lugar de destino, los gobiernos de los lugares emisores-receptores y las 
comunidades locales de acogida de visitantes temporales en un destino diferente a su lugar de residencia habitual.

Según De Velasco (2009), "Gestión es un término aplicado a una serie de herramientas, el uso adecuado de estas determinará la eficacia de dicha gestión. Tradicionalmente se asociaba gestión con dirección o mando".

Entendemos por gestión el manejo integral de un proyecto o de un proceso de planificación. Para Debreczein (2003), "La gestión implica, control total del desenvolvimiento de sus componentes, seguimiento de las actividades programadas, medición del logro de objetivos propuestos y la readecuación a las condiciones cambiantes".

Según Pearce (2016), "La diversidad de modelos que existe es una característica de una literatura emergente en la cual todavía no hay conceptos y modelos dominantes, y los investigadores siguen explorando numerosos caminos distintos".

Para Bercial y Timón (2005), "El turismo debe ser gestionado en el espacio geográfico por lo tanto debe trabajarse desde tres grandes marcos conectados entre sí: los aspectos sociales del desarrollo, elementos de la sostenibilidad ambiental y territorial y el abordaje de los aspectos económicos"

De ahí que para la investigación es trascendental la comunicación con propietarios o gerentes de hoteles, porque partiendo de su realidad se obtiene la información necesaria para planificar esta base de la gestión y darle una nueva visión al destino Coca.

Según Moreno et al. (2018), "Los procesos de planificación y gestión de destinos están en la base del desarrollo turístico de los destinos que resultan necesarios para ordenar los procesos de desarrollo en función de las prioridades del territorio y de su población local”.

Para Frangialli (1999), "La diversificación es el camino ante mercados cada vez más segmentados tanto en motivaciones como en variables sociodemográficas y económicas, por ello surgen ofertas que más bien compiten con el precio y son obstáculos para la rentabilidad".

Según Rodriguez (2005), "La tendencia de los últimos 5 años muestra que el turismo se ha convertido en una importante actividad económica que ha confirmado, a través del tiempo, su capacidad para sortear las crisis económicas de las economías subdesarrolladas".

El caso del destino Ecuador que presenta una influyente crisis y debilidad económica en el período de investigación, supone que su tendencia se direcciona en adaptar los gustos y demandas de los consumidores, especialmente en el turismo ya que es una estrategia socioeconómica para dinamizarse.

Ribeirinho (2010), afirma que "El turista de la era del conocimiento y la información es un consumidor exigente, para quien, el tiempo de ocio forma parte esencial de su 
autorrealización personal y social”.

El turismo internacional ha pasado de ser una pequeña industria para transformarse en un imperio de operaciones que cambian constantemente, influenciado por el uso de la tecnología. Riveros (2013), menciona que "La actividad turística se ha transformado en uno de los sectores económicos con mayor envergadura y crecimiento del mundo".

Según la Organización Mundial del Turismo OMT (2018), "El crecimiento de las llegadas de turistas internacionales se manifiesta en un 3.32\% anualmente y la previsión para los años 2010 al 2030 se llegaría a los 1.800 millones de llegadas de turistas internacionales a escala mundial". De lo anterior se comprende que aun cuando el mundo percibe distintas conmociones de índole social, natural o económicas que pueden afectar negativamente los destinos internacionales, su adaptabilidad le representa una fortaleza que es producto de su evolución como industria y pilar de la dinamización económica mundial.

Según el Ministerio del Ambiente (2016), "Ecuador se encuentra en el sexto puesto de 17 países megadiversos del planeta, y es uno de los países donde se concentra la mayor biodiversidad del planeta", se suma su multiculturalidad con 13 nacionalidades y otros 13 pueblos dentro de la nacionalidad kichwa.

El cantón Francisco de Orellana tradicionalmente conocido como El Coca cuenta con una infraestructura variada en cuanto al alojamiento, alimentos y bebidas, bares, agencias de viajes entre otros, en total existen 52 establecimientos hoteleros registrados legalmente que se encuentran catastrados y que son los actores del desarrollo turístico del Coca.

En cuanto al sector petrolero, Ecuador figura entre los países que fueron más beneficiados por el boom petrolero de los setenta. Según Fontaine (2013), "El crecimiento disminuyó en los ochenta agudizándose en un 2.1\% anualmente y para 1988 el PIB del estado decreció en un $10.5 \%$ resultado de la crisis y dependencia de la economía nacional al sector petrolero".

Debido a que el petróleo es prioridad las cifras de la bonanza son muy altas, pero enmascaran importantes debilidades estructurales y es más profundo el pozo en el que se está dejando caer la economía del Coca.

Según Pástor (2020), "Entre el 2020 y 2025, la demanda mundial de petróleo se estima crecerá, tomando en cuenta también el fuerte incremento de la demanda asiática de petróleo, creando así oportunidades para que los países productores de petróleo también incrementen sus exportaciones".

\section{Planteamiento metodológico}

El enfoque cualitativo permitió indagar, describir y comprender una realidad concreta, vinculada con la incidencia del sector petrolero en la actividad turística de la ciudad del Coca durante un período de tiempo que está comprendido entre el año 2010-2015 cuando existió una bonanza económica y el periodo 2016-2019 en el mayor declive económico percibido por su población. 
Además, se avanzó en el análisis de las percepciones sobre la dependencia del turismo al desarrollo petrolero en el área de análisis, tanto desde la perspectiva de los gerentes y dueños de establecimientos hoteleros.

A partir de este aspecto puede destacarse que el diseño de la investigación fue de carácter descriptivo y analítico-sintético, porque consistió en la caracterización de un hecho, fenómeno, individuo o grupo, con el fin de establecer su estructura o comportamiento.

Se utilizó el método analítico - sintético mediante el cual se diferenciaron los datos de las entrevistas, se integraron los criterios recurrentes tanto de carácter cualitativo como cuantitativo, se analizó la información y se sintetizó los resultados en tablas, gráficos.

Para la presente investigación se seleccionó una unidad de análisis con funcionamiento desde el año 2010 hasta el año 2019 y que se encuentra activa:

1. Establecimientos hoteleros en la ciudad del Coca.

En relación con esta unidad de análisis se seleccionó la unidad de revisión:

1. Gerentes y/o dueños de establecimientos hoteleros.

Con respecto al análisis de la información del sector petrolero en cuanto a volúmenes de producción e ingresos percibidos durante el periodo 2010 -2019 se consultó los boletines emitidos por el Banco Central del Ecuador. Al tratarse de datos cuantitativos de tipo continuo, se utilizó medidas de promedios y de tendencia central. Para la determinación del coeficiente de correlación se calculó el de Pearson, que es el que corresponde a este tipo de datos.

La población total estuvo constituida por 52 establecimientos hoteleros de la ciudad del Coca. El tipo de muestreo fue intencional no probabilístico, ya que "es un método empleado para estudiar un individuo o una institución, en un entorno o situación única, y de una forma lo más intensa y detallada posible" García (2005).

Las entrevistas realizadas fueron no probabilísticas, ya que la elección de los elementos no depende de la probabilidad sino de causas relacionadas y dependen de la capacidad operativa de recolección y análisis, así como también de la predisposición de los referentes de cada uno de los establecimientos para ser indagados, aportando información y datos relevantes para la investigación.

Se tuvo como herramienta de recolección de datos a la entrevista y la técnica es el cuestionario a profundidad semiestructurado para lo cual se consideraron los ítems que se detallan a continuación:

1. Delimitación espacial: Ciudad del Coca

2. Involucrados: Establecimientos de servicios hotelero en la ciudad del Coca.

3. Información proporcionada: Depende del involucrado, sobre características de cada hotel, situación actual, experiencias, vivencias, formas de pensar, aptitudes y actitudes, motivaciones, objetivos, capacidades, formas 
de trabajo, procedimientos, políticas, percepciones, puntos fuertes y débiles, amenazas y oportunidades, reacciones.

4. Tiempo: La duración de las entrevistas deben tener en promedio 27 minutos, debido a que se realizó una prueba piloto durante la cual se comprobó que en promedio ese es el tiempo suficiente para contestar el cuestionario semiestructurado.

5. Validez de aplicación: Se funda en la confianza que se transmita al entrevistado de la cual se obtuvieron anotaciones objetivas.

6. Confiabilidad: Se documentaron las grabaciones de las entrevistas y se incluyeron los datos en una matriz. Según recomienda Scribano (2008), se tomó nota de todo lo sucedido en el entorno y de todo lo que los informantes hacen y dicen.

7. Validación: Triangulación entre fuentes de información secundaria y datos relevantes.

\section{Resultados}

El sector petrolero inició su desarrollo el 29 de marzo de 1967 cuando empezó a extraer del primer pozo en la Amazonía del Ecuador, el LAGO AGRIO 1 de la Compañía TEXACO GULF produciendo 2.610 barriles diarios, aquí se inició la era petrolera ecuatoriana.

Según Sigüenza y Silva (2007), "Existen tres filiales permanentes de Petroecuador que son (Petroproducción, Petrocomercial y Petroamazonas), al respecto la filial de Petroproducción trabaja en el norte de la Amazonía y específicamente en Orellana, sus principales campos son el SACHA y AUCA", (Ver figura 1): que tiene los subcampos Sacha, Pucuna, Payamino, Paraíso, Big Uno, Huachi, Mauro Dávalos y Coca entre los más explotados, también existe una producción compartida con empresas petroleras privadas como el subcampo Coca - Payamino.

\section{Figura 1}

Pto. Francisco de Orellana, campos de petróleo catastrados

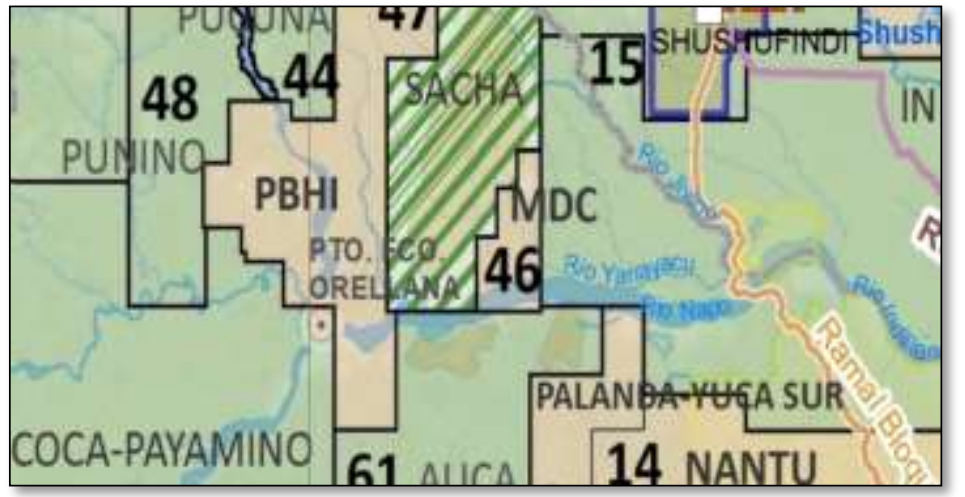

Fuente: http://www.eppetroecuador.ec/wp-content/uploads/downloads/2015/03/MapaCatastral.jpg

Referente al comportamiento del precio promedio anual del barril de petróleo el reporte del sector petrolero del Banco Central del Ecuador destaca en el año 2010 un precio de 
\$72,33 USD que tiende a subir hasta el año 2012 a \$98,92 USD e inicia su caída desde el año 2013 hasta el 2016 cuando se reporta un precio de venta promedio anual de \$36,98 USD, precio que responde a un sistema internacional de regularización del precio que favorece a las transnacionales petroleras y no a los pequeños países como Ecuador al momento de exportar su crudo. A partir de entonces hasta el año 2019 se aprecia un ligero incremento al 2018 y nuevamente cae en el 2019 a \$ 58.38 USD, tal como se aprecia en la figura 2.

\section{Figura 2}

Precio promedio anual del barril de petróleo ecuatoriano. Periodo 2010-2019

\section{PRECIO PROMEDIO ANUAL DEL BARRIL DE PETROLEO ECUATORIANO}

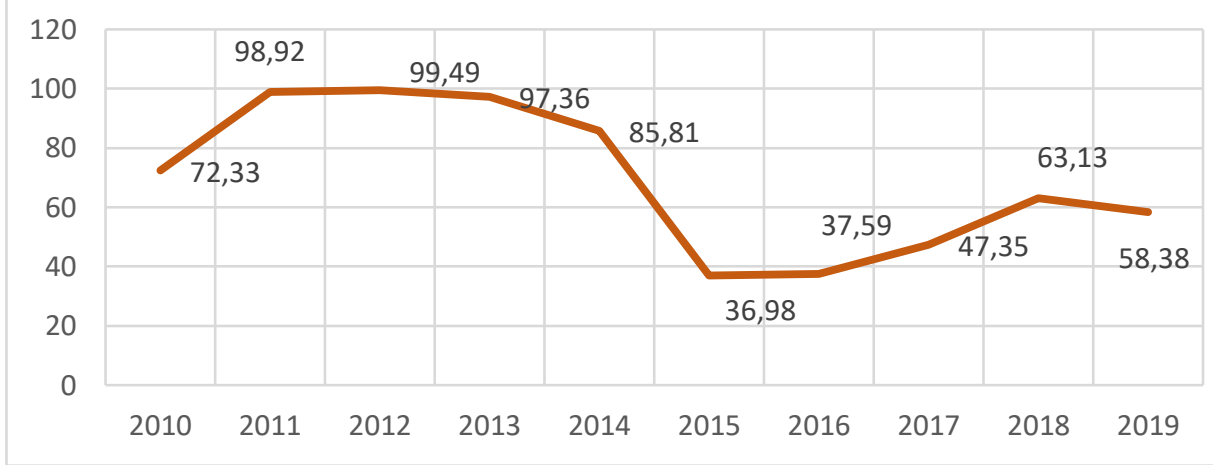

Fuente: Elaboración propia a partir de los datos del Banco Central del Ecuador

En la figura 3 se observa las variaciones del volumen de crudo en millones de barriles exportados por el Ecuador desde el año 2010 al 2019, según los reportes anuales de EP PETROECUADOR se han exportado entre 120 y 155 millones de barriles de petróleo como la cifra más alta del período de investigación, el comportamiento del año 2010 inicia con 124,5 millones lo cual desciende en el 2011 a 121,7 millones, pero aumenta la exportación en el año 2012 hasta el pico más alto que fue en el año 2014 cuando se logró exportar 154,7 millones de barriles, el año 2015 y 2018 descendió la cifra hasta 129,69 y a finales del año 2019 se percibe un leve incremento, hasta 139.82

Figura 3

Volumen de barriles exportados (Millones Bls). Periodo 2010-2019

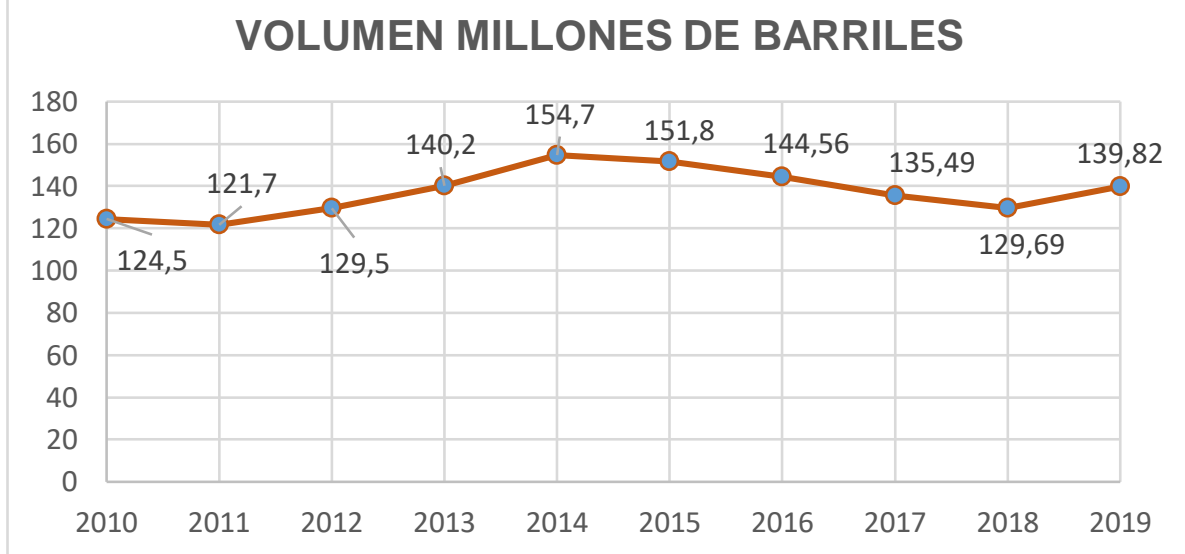

Fuente: Banco Central del Ecuador. Reporte del sector petrolero 2019 
La fusión del indicador volumen en millones de barriles exportados y el indicador precio de venta para exportación confluyen en el valor de las exportaciones del país en millones de dólares como se muestra en la figura 4 , en donde se observa un incremento desde el año 2010 al 2013 cuando llega a la cumbre con 13411,8 millones de dólares y empieza su reducción en el año 2014 con 13016 millones de dólares, hasta caer en el año 2016 en 5053,94 millones de dólares. En los años 2017 y 2018 se observa un leve crecimiento, pero al 2019 otra vez se presenta un decaimiento con 7731.17 millones de dólares. Dólares. (Banco Central del Ecuador, 2020)

\section{Figura 4}

Exportaciones Nacionales en Millones USD

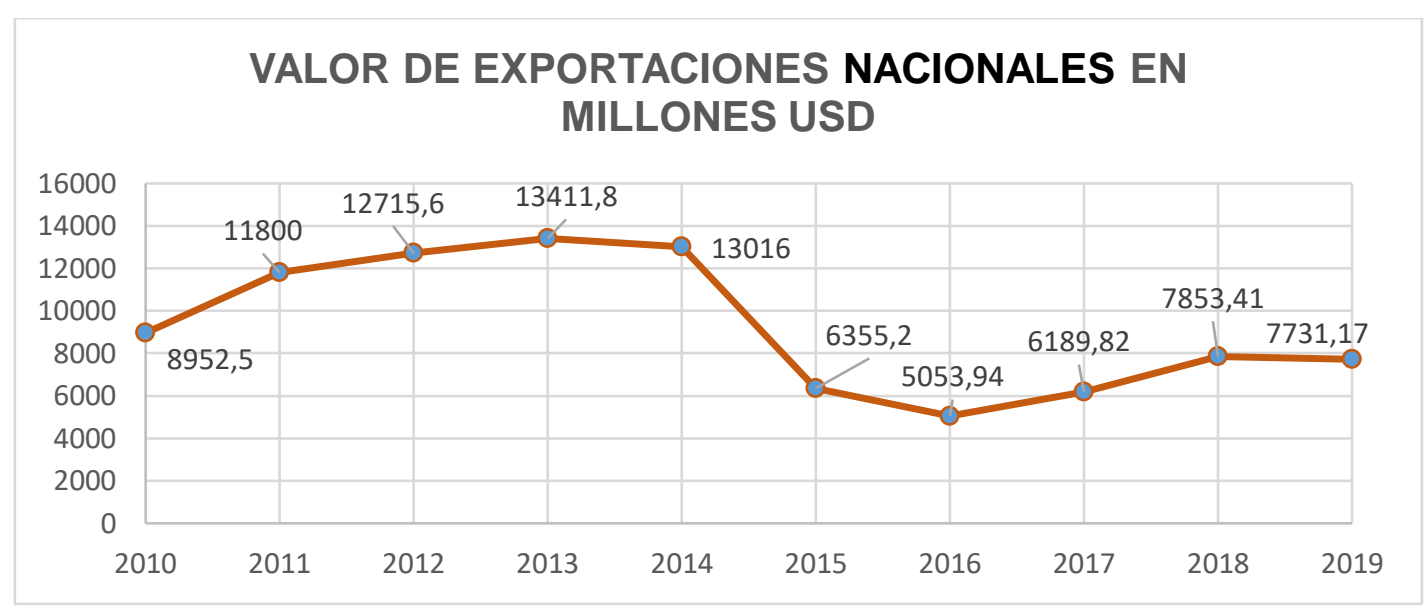

Fuente: Banco Central del Ecuador. Reporte del sector petrolero 2019

\section{Caracterización de los establecimientos hoteleros del Coca}

Se detalla los principales hoteles del Coca

Tabla 1

Oferta hotelera de Francisco de Orellana

\begin{tabular}{|c|c|c|c|c|c|}
\hline No. & NOMBRE & CATEGORÍA & & NOMBRE & CATEGORÍA \\
\hline 1 & Karen & segunda & 27 & Chambira & tercera \\
\hline 2 & Cisne el & tercera & 28 & Amazonas & tercera \\
\hline 3 & Gran hostal paraíso amazónico & tercera & 29 & $\begin{array}{l}\text { Vista } \\
\text { amazónica en } \\
\text { Payamino }\end{array}$ & tercera \\
\hline 4 & El oro & tercera & 30 & Amazónico & tercera \\
\hline 5 & El Marquéz & primera & 31 & $\begin{array}{l}\text { Gran hotel del } \\
\text { Coca C.P. }\end{array}$ & primera \\
\hline 6 & Damaris & tercera & 32 & $\begin{array}{l}\text { Heliconias } \\
\text { gran hotel }\end{array}$ & segunda \\
\hline 7 & Puerto orellana & segunda & 33 & $\begin{array}{l}\text { Gran hotel } \\
\text { bosque } \\
\text { dorado }\end{array}$ & tercera \\
\hline 8 & Río napo & segunda & 34 & Jekannyty & segunda \\
\hline 9 & Canoas & tercera & 35 & Zuria & tercera \\
\hline 10 & San Luis & tercera & 36 & La misión & primera \\
\hline 11 & López & tercera & 37 & El Auca & primera \\
\hline 12 & Nuevo amanecer & tercera & 38 & Cabaña & tercera \\
\hline
\end{tabular}


Tabla 1

Oferta hotelera de Francisco de Orellana (continuación)

\begin{tabular}{llllll}
\hline No. & NOMBRE & CATEGORÍA & & NOMBRE & CATEGORÍA \\
\hline 13 & Ciudad canela & segunda & 39 & Caracas suite & tercera \\
14 & Casa blanca & tercera & 40 & Omaguas & tercera \\
15 & Lojanita & tercera & 41 & Yasuní 2 & tercera \\
16 & Santa maría & tercera & 42 & Coca imperial & tercera \\
17 & Coca & tercera & 43 & Shaddai & segunda \\
& & & & Gran hotel & \\
18 & Unicornio & tercera & 44 & perla & tercera \\
& & & & amazónica & \\
19 & Williams & tercera & 45 & Gran hotel rey & tercera \\
20 & safari brisas del napo & tercera & 46 & Yasuní & tercera \\
21 & Henry Paúl & tercera & 47 & Cotopaxi & cuarta \\
22 & San Fermín & tercera & 48 & Orellana & tercera \\
23 & Florida & tercera & 49 & La luna & tercera \\
24 & Oasis & tercera & 50 & Paraíso & tercera \\
25 & Hostería amazonas & tercera & 51 & La torre & tercera \\
26 & La curva del cañón & tercera & 52 & Royal Palace & tercera \\
\hline
\end{tabular}

Fuente: Elaboración propia a partir del Registro de catastros de los establecimientos hoteleros de Francisco de Orellana

\section{Hotel el Auca}

El hotel El Auca se inició como un campamento que prestaba los servicios de alojamiento exclusivamente para los trabajadores de la TEXACO (Texas Petroleum Company). Fue uno de los primeros hoteles que se creó la ciudad del Coca, formalmente se registró su apertura el 24 de noviembre de 1973, su ubicación es en la Calle Napo 31-01 entre García Moreno y Rocafuerte, centro económico de la urbe.

Su gerente manifiesta que no posee un organigrama estático sino más bien una estructura de procesos en cada área. Oferta a más del alojamiento, el servicio de restaurante, sala de eventos, y spa. Se ha enfocado en un segmento del mercado nacional e internacional con una capacidad de gasto medio y/o alto, la mayor parte de sus huéspedes son adultos profesionales de las diferentes áreas del sector público y empresas privadas, el tiempo de su estadía en promedio es de 2 a 3 días, complementariamente arriendan habitaciones mensualizadas a los trabajadores del sector petrolero.

Su publicidad la realiza a través de su página web y demás medios electrónicos, también se promociona en Facebook donde presenta una página interactiva para reservas y novedades en sus servicios. Su capacidad es de 74 habitaciones con 90 plazas y 82 mesas con 382 plazas.

Los detalles del interior de la recepción brindan un ambiente amazónico que promueve la aventura en el Parque Nacional Yasuní. Es evidente la organización administrativa del establecimiento hotelero con la presentación a los huéspedes de su misión, visión y política de calidad, y se evidencia el izamiento de las banderas de países de la región y Sudamérica como parte de la hospitalidad del hotel. 
El hotel presenta una gestión integral, cuenta con sus logos promocionales, esta integralidad se acopla a las políticas de las entidades públicas que colaboran en el territorio para el surgimiento de las ventas. La capacitación al personal es constante en el turismo, que se corrobora con certificaciones que le acreditan como: la $\mathrm{Q}$ de calidad que otorgó el Distrito Metropolitano de Quito en el 2016 y el certificado de Oro de la empresa asociada a la ISO.

\section{Figura 5}

Hotel El Auca. Fachada frontal

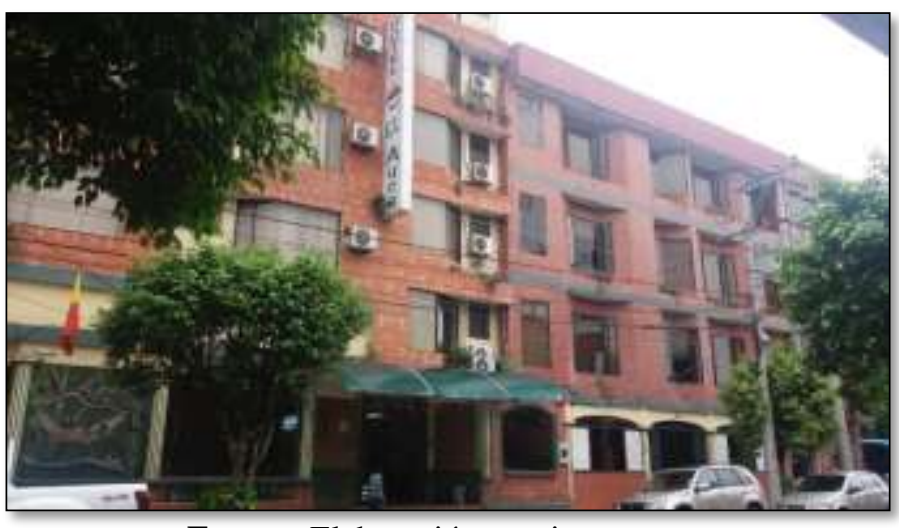

Fuente: Elaboración propia

El porcentaje de incidencia del desarrollo petrolero es de $-31.25 \%$, tomando en cuenta la diferencia entre el promedio de temporada de bonanza y de recesión.

\section{Tabla 2}

Incidencia del desarrollo petrolero en la actividad hotelera del Coca

\begin{tabular}{lccc} 
ACTIVIDAD & $\begin{array}{c}\text { Promedio temporada } \\
\text { de bonanza }\end{array}$ & $\begin{array}{c}\text { Promedio temporada } \\
\text { de recesión }\end{array}$ & $\begin{array}{c}\% \text { de } \\
\text { INCIDENCIA }\end{array}$ \\
\hline Alojamiento & 16896 & 11616 & $-31.25 \%$ \\
\hline
\end{tabular}

Fuente: MINTUR (2020)

Figura 6

Incidencia del desarrollo petrolero en la actividad hotelera del Coca

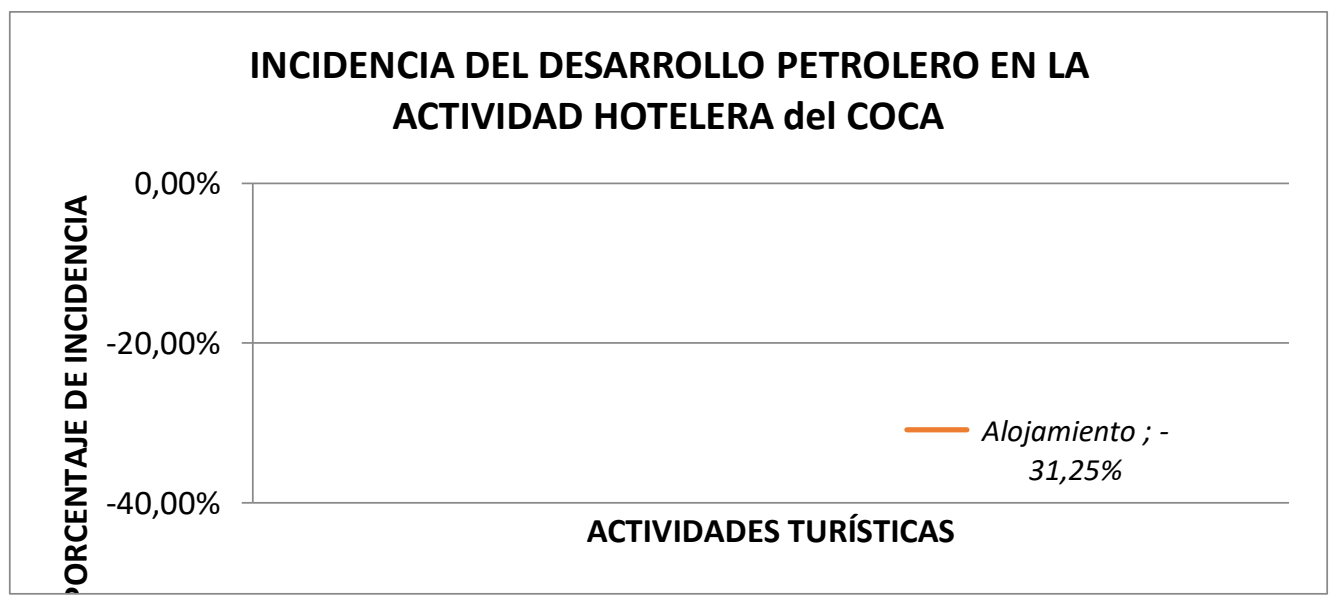

Fuente: MINTUR (2020) 
Tabla 3

Ocupación del segmento Petrolero en los hoteles del Coca según el período de análisis

\begin{tabular}{ccccccccccc}
\hline DEMANDA & $\mathbf{2 0 1 0}$ & $\mathbf{2 0 1 1}$ & $\mathbf{2 0 1 2}$ & $\mathbf{2 0 1 3}$ & $\mathbf{2 0 1 4}$ & $\mathbf{2 0 1 5}$ & $\mathbf{2 0 1 6}$ & $\mathbf{2 0 1 7}$ & $\mathbf{2 0 1 8}$ & $\mathbf{2 0 1 9}$ \\
\hline Petrolera & $55 \%$ & $59 \%$ & $65 \%$ & $67 \%$ & $70 \%$ & $55 \%$ & $40 \%$ & $58 \%$ & $61 \%$ & $57 \%$
\end{tabular}

*El año 2014 fue el año de mejor ocupación del segmento petrolero para el sector hotelero del Coca. Fuente: AHOTEC (2020)

La tabla 3 indica que la demanda del segmento petrolero va aumentando porcentualmente desde el año 2010 hasta el año 2014 cuando se evidencia un 70\% de ocupación, coincidiendo con el mejor año de ocupación hotelera en El Coca. Por otra parte, en el año 2016 la ocupación cayó abruptamente al 40\%, lo cual supuso una crisis en la actividad turística de hospedaje. En los años 2017 al 2019 se observa un incremento que promedia el $18 \%$, sin llegar a los porcentajes alcanzados entre el 2012 - 2014. Una razón por la cual el segmento corporativo petrolero dejó de hospedarse en los hoteles del Coca fue la modalidad de campamentos en el interior de las empresas y pozos que readaptaron las compañías de petróleo para reducir sus gastos y optimizar las ganancias de la empresa, en dichos campamentos el hospedaje y la alimentación forman parte del contrato laboral, este cambio se dio con más fuerza en el año 2016 cuando el precio del barril del petróleo por segundo año consecutivo tuvo un promedio diario inferior a los 40 dólares y las exportaciones de crudo estuvieron a la baja, otro argumento es el cierre de compañías de petróleo y el consecuente despido del personal administrativo y trabajadores relacionados directamente a la industria petrolera.

\section{Tabla 4}

Ingreso de visitas al Parque Nacional Yasuní. Años 2010-2019

\begin{tabular}{lc}
\hline AÑO & TOTAL \\
\hline 2010 & 4150 \\
2011 & 10313 \\
2012 & 8486 \\
2013 & 9860 \\
2014 & 13876 \\
2015 & 12203 \\
2016 & 11090 \\
2017 & 11722 \\
2018 & 13159 \\
2019 & 11213 \\
\hline Fuente: SIB (2020)
\end{tabular}


Figura 7

Ingreso turistas al Parque Nacional Yasuní

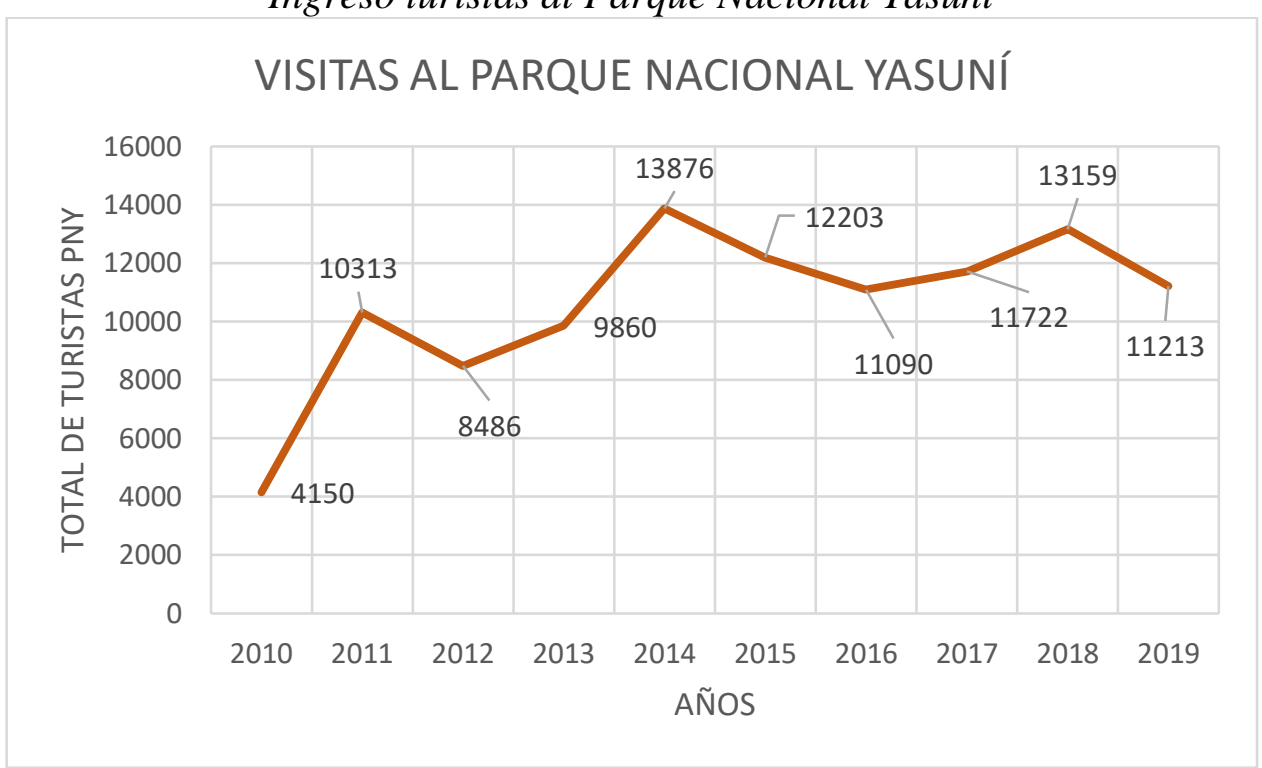

Fuente: Estadísticos SIB 2010-2019 - PNY (1).pdf

En la tabla 4 y figura 7, es notorio el incremento en el ingreso de turistas en el año 2011, más extraordinariamente en el año 2014 con 13876 visitas y el 2015 cuando la recesión económica fue evidente en el país, se registró también un alto número de turistas con un ingreso de 12.203 de los cuales el $76.94 \%$ corresponde a extranjeros y su diferencia son nacionales SIB (2020).

\section{Figura 8}

Impacto del desarrollo petrolero en la actividad hotelera del Coca

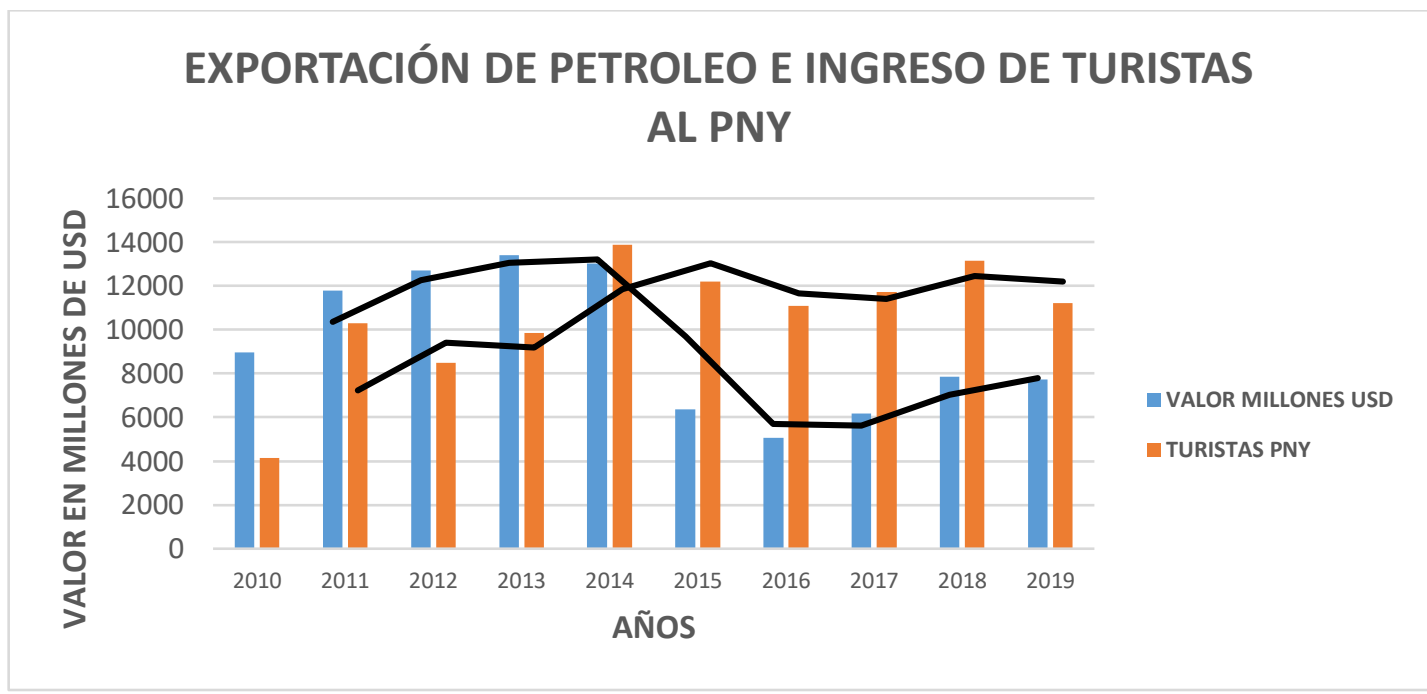

Fuente: Banco Central del Ecuador (2020) y SIB (2020)

En la figura 8, por una parte, se observa la campana de las exportaciones de petróleo donde es notoria la caída del año 2015 y más en el 2016, mientras que por otra parte se evidencia el incremento de turistas excepto en el descenso del año 2016, notándose en los años subsiguientes una ligera recuperación hasta el año 2018 y que nuevamente tiende a 
la baja en el año 2019. Parte la reducción de turistas para el año 2016 responde a la necesidad de resguardar su economía familiar reduciendo el gasto en turismo, esto sumado al factor del terremoto de abril del 2016 en la costa ecuatoriana que a su vez influyó en la cancelación de reservaciones y viajes hacia el Ecuador y por ende hacia el PNY, por lo que se justifica este decrecimiento de turistas en la ciudad del Coca.

Figura 9

Impacto del desarrollo petrolero vs ocupación hotelera del segmento petrolero del Coca

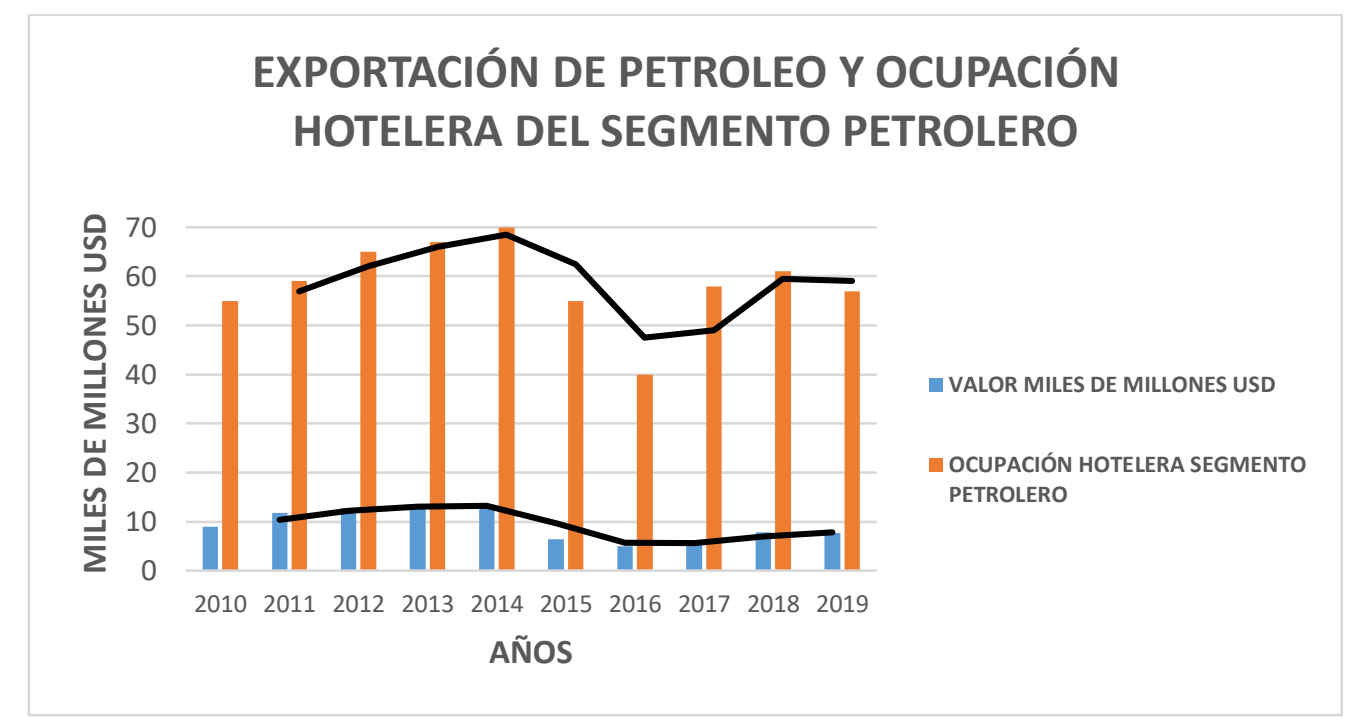

Fuente: Banco Central del Ecuador (2020) y AHOTEC (2020)

En la figura 9 se observa que la campana de las exportaciones se comporta de manera similar a la campana de ocupación hotelera del segmento petrolero. La tendencia incremental se manifiesta hasta el año 2014, mientras que en los años 2015 y 2016 decaen ambas campanas, para recuperarse luego entre el año 2017 y 2019.

Se procedió a evaluar la correlación entre el desarrollo petrolero en el periodo 2010 -2019 y la actividad hotelera (tomando como referencia el número de turistas que ingresaron al PNY.

Por tratarse de datos cuantitativos continuos, el grado de correlación se determinó utilizando el coeficiente de Pearson.

Tabla 5

Medidas de tendencia central para exportación petrolera y turistas PNY

\begin{tabular}{lccc}
\hline & Media & Desviación. Std & $\mathrm{N}$ \\
\hline $\begin{array}{l}\text { Exportación petróleo } \\
\text { millones usd }\end{array}$ & 9307.94 & 3156.64 & 10 \\
Turistas PNY & 10607.20 & 2757.33 & 10 \\
\hline
\end{tabular}

Fuente: Banco Central del Ecuador (2020) y SIB (2020) 
Tabla 6

Correlación de Pearson: Exportaciones de petróleo e ingreso de turistas al PNY

\begin{tabular}{llc} 
& & $\begin{array}{c}\text { Turistas } \\
\text { PNY }\end{array}$ \\
\hline $\begin{array}{l}\text { Exportación petróleo } \\
\text { millones usd }\end{array}$ & Correlación de & -.147 \\
& Pearson & .685 \\
& Sig. (2-colas) & 10 \\
\hline
\end{tabular}

Prácticamente no existe correlación entre las exportaciones nacionales de petróleo y el ingreso de turistas al Parque Nacional Yasuní. El valor de 0.147 es tan bajo que se lo puede considerar marginal. Este resultado explica que la ocupación hotelera por parte de turistas se comportó sin afectación de la crisis del petróleo ecuatoriano, puesto que los visitantes al PNY en su mayoría son extranjeros, ajenos a la crisis ecuatoriana.

\section{Tabla 7}

Medidas de tendencia central para exportación petrolera y ocupación hotelera del sector petrolero

\begin{tabular}{lccc}
\hline & Media & Desviación. Std & $\mathrm{N}$ \\
\hline $\begin{array}{l}\text { Exportación de petróleo } \\
\text { miles de millones usd }\end{array}$ & 9.30 & 3.15 & 10 \\
$\begin{array}{l}\text { Ocupación segmento } \\
\text { petrolero }\end{array}$ & 58.70 & 8.31 & 10 \\
\hline
\end{tabular}

Fuente: Banco Central del Ecuador (2020) y AHOTEC (2020)

\section{Tabla 8}

Correlación de Pearson: Exportaciones de petróleo vs ocupación hotelera del segmento petrolero

\begin{tabular}{|c|c|c|}
\hline & & $\begin{array}{l}\text { Ocupación segmento } \\
\text { petrolero }\end{array}$ \\
\hline \multirow[t]{3}{*}{$\begin{array}{l}\text { Exportación de petróleo } \\
\text { miles de millones usd }\end{array}$} & $\begin{array}{l}\text { Correlación de } \\
\text { Pearson }\end{array}$ & $.810^{* *}$ \\
\hline & Sig. (2-colas) & .004 \\
\hline & $\mathrm{N}$ & 10 \\
\hline
\end{tabular}

Se encontró una asociación lineal estadísticamente significativa, alta y directamente proporcional $\left(\mathrm{R}_{\mathrm{P}}=0.810, \mathrm{p}=0.004\right)$, entre las exportaciones nacionales de petróleo (miles de millones usd) y la ocupación hotelera de trabajadores del sector petrolero. 
Figura 10

Impacto de las exportaciones nacionales de petróleo en la ocupación hotelera de trabajadores del sector petrolero

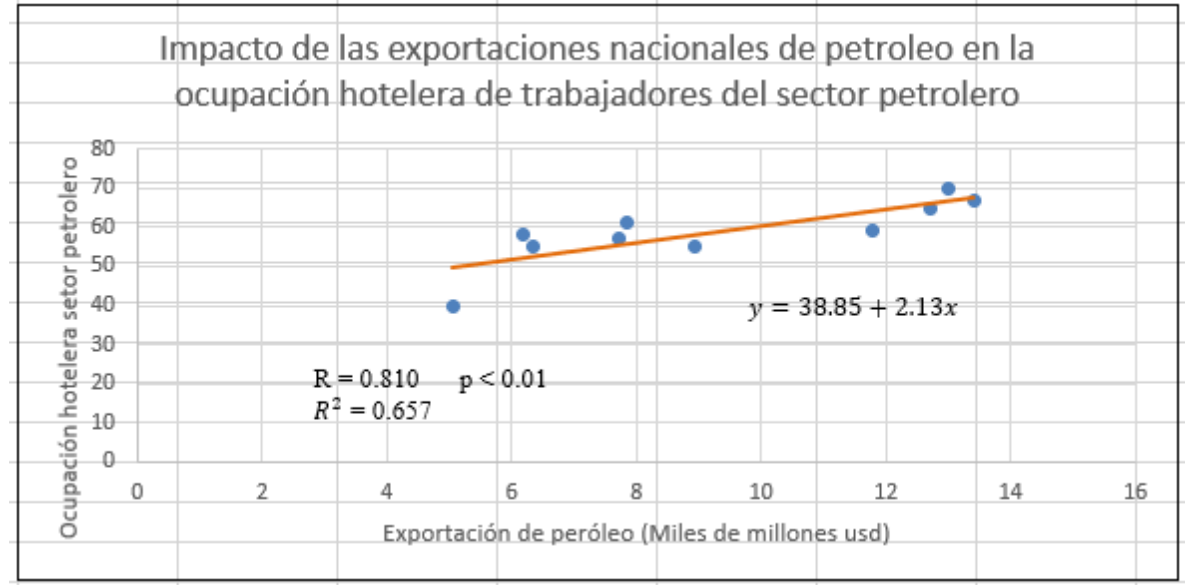

El coeficiente de determinación $\left(\mathrm{R}^{2}\right)$ es igual a 0.657 lo que significa que, el $65.7 \%$ de la variación total de la variable "Ocupación hotelera de trabajadores del sector petrolero" es explicada por la variable "Exportación nacional de petróleo" en el modelo de regresión. La ecuación $(y=38.85+2.13 x)$, permitiría predecir la ocupación hotelera en función de las exportaciones petroleras del país.

\section{Conclusiones}

- Se caracterizaron los establecimientos turísticos de hospedaje, encontrando que en medio de la diversidad presentan rasgos de similitud al respecto de su formación empírica que de a poco ha ido profesionalizándose principalmente en los establecimientos de primera categoría, se identificó una tendencia hacia el segmento de mercado corporativo petrolero debido a su alta capacidad de gasto; la gestión de forma general no es clara y en especial los establecimientos de segunda y tercera categoría no presentan objetivos estratégicos definidos y actualizados, mucho menos una misión y visión acorde a la realidad después de la recesión económica en El Coca.

- El precio del barril de petróleo fue cambiante, en el 2015 y 2016 se tuvo el piso económico llegando a \$36,98 USD y luego tuvo un ligero incremento, precio que incide directamente en el PIB del estado y su solvencia, concluyendo que existió una recesión económica en el año 2016. Algunas empresas petroleras que se desarrollaron entre los años de bonanza optaron por contratar los servicios de hospedaje en el Coca, lo cual generó una bonanza económica en las actividades turísticas hasta el año 2014, pero luego genero una fuerte reducción de las ventas en el año 2016 cuando el precio del barril del petróleo cayó.

- La incidencia del desarrollo del sector petrolero en la actividad turística del Coca trajo consigo una época de bonanza económica entre el año 2010 al 2014 que logró copar los hoteles. La época de recesión entre el 2015 y 2016 perjudicó al sector turístico por varios factores como: la mono segmentación del mercado al cual se dedicó, una realidad internacional de cautela en el gasto en turismo, 
despido de los empleados de las empresas de petróleo tanto públicas y privadas, cierre de los emprendimientos afines al sector turístico.

- Se evidenció que existe una relación de dependencia altamente significativa entre los ingresos petroleros y la demanda hotelera del segmento petrolero ( $\mathrm{sig}=0.004$ ). El coeficiente de correlación de Pearson alcanzó un valor de 0.810 que evidencia una alta correlación lineal positiva entre las exportaciones de petróleo y la demanda hotelera por parte del segmento petrolero en la ciudad del Coca.

\section{Referencias bibliográficas}

AHOTEC. (2020). Ocupación hotelera en el Coca. Coca: AHOTEC.

Alcaldía de Francisco de Orellana. (6 de Agosto de 2019). Plan de Desarrollo y Ordenamiento Territorial Municipal de Francisco de Orellana 2014-2019. Obtenido de https://orellana.gob.ec/docs/PDyOT\%20GADMFO\%2020142019.pdf

Ander-Egg, E. (1982). Técnicas de investigación social. Buenos Aires: Humanitas.

Banco Central del Ecuador. (2020). Reporte del Sector Petrolero. Quito: BCE.

Bercial, R., \& Timón, D. (2005). Nuevas tendencias en el desarrollo de destinos turísticos: marcos conceptuales y operativos para su planificación y gestión. Cuadernos de turismo.

Caro, J., Luque, A., \& Zayas, B. (2015). Nuevas tecnologías para la interpretación y promoción de los recursos turísticos culturales. Revista de turismo y patrimonio cultural.

Casallas, J. (2014). El emprendimiento y su incidencia en el desarrollo socioeconómico del Casanare desde el sector petrolero. Bogotá: Trabajo de grado. Universidad Militar Nueva Granada.

De Velasco, J. (2009). Gestión por procesos. México: Esic Editorial.

Debreczein, E. (2003). Gestión del turismo sostenible y patrimonio cultural. Portal Iberoamericanos de gestión cultural.

EPPETROECUADOR. (2016). Memoria de sostenibilidad. Obtenido de http://www.eppetroecuador.ec/wpcontent/uploads/downloads/2016/10/Memoria-EP-Petroecuador.pdf

Fontaine, G. (2013). Sobre bonanzas y dependencia: petróleo y enfermedad holandesa en el Ecuador. Íconos - Revista de Ciencias Sociales, 102-110.

Frangialli, F. (1999). Sustainable Tourism. Madrid: WTO (OMT). 
García, M. (2005). Metodología de investigación científica. USN. Métodos de estudio de caso. Obtenido de http://www.itescham.com/Syllabus/doctos/r1614.DOC

Goeldner, C., \& Ritchie, B. (2012). Tourism: Principles,Practices, philosophies. Canada: Wiley\&Sons.

Gutiérrez, C. (2013). La resiliencia como factor clave en la recuperación de destinos turísticos. Valencia: Universidad de Valencia. Tesis dostoral.

Guzmán-Sala, A. (2016). Innovations dans le sectour toristique aи Mexique. Paris: L'Harmattan.

Martín, R. (2003). Manual de teoría y práctica del turismo. Obtenido de http://intranet.uclv.edu.cu/fiit/cetur

Ministerio del ambiente. (2016). Áreas protegidas del Ecuador. Quito: Ministerio del ambiente.

Ministerio de Turismo (2020). Hagámosle fácil. Obtenido de:

https://www.turismo.gob.ec/informe-de-rendicion-de-cuentas/

Moreno, A., Sariego, I., \& Reyes, B. (2018). La planificación y la gestión como herramientas de desarrollo de los destinos turísticos. TURyDES, 4-7.

OMT. (2018). Panorama OMT del Turismo internacional. Madrid: OMT.

Page, S. (2009). Tourism management managing for change. Oxford: Elsiever.

Pástor, S. (4 de Junio de 2020). Perspectivas petroleras y su impacto en el Ecuador y en los GAD. Obtenido de http://www.congope.gob.ec/wpcontent/uploads/2020/06/Perspectivas-Petroleras-Ecuador-GAD.pdf

Pearce, D. (2016). Modelos de gestión de destinos. Síntesis y evaluación. Vol 25, № 1. Estudios y perspectivas en turismo, 1-5.

Ribeirinho, J. (2010). Turismo, fundamentos conceptuales, realidad y perspectivas. Navarra: Universidad de Navarra.

Riveros, H. (2013). El agroturismo, una alternativa para revalorizar la agroindustria rural como mecanismo de desarrollo local. Lima: IICA-PRODAR.

Rodríguez, R. (2005). Gestión de destinos turísticos. Texto docente Centro de Estudios Turísticos. Universidad de la Habana. Obtenido de www.uclv.edu.cu/biblioteca

Sancho, A. (5 de Agosto de 2021). Definición de turismo. Obtenido de Que es turismo: https://wiki.ead.pucv.cl/index.php/Definici\%C3\%B3n_de_turismo__Conoce_Valpara\%C3\%ADso 
Scribano, A. (2008). El proceso de investigación social cualitativo. Buenos Aires: Prometeo.

SIB. (2020). Estadísticos SIB 2010-2019 - PNY. pdf. Quito: Ministerio del Ambiente.

Sigüenza, F., \& Silva, R. (2007). El petróleo ecuatoriano y su incidencia en la economía nacional. Cuenca: Tesis Economía. Universidad de Cuenca.

Tafur, G., Vélez, C., Alejo, O., Zumba, M., \& Jácome, J. (2018). Desarrollo tecnológico del sector turístico en Guayaquil - Ecuador. Espacios, 3.

Valls, J. (2004). Gestión de destinos turísticos sostenible. España: Ediciones gestión.

Visión 360. (11 de Agosto de 2021). Estado deudor. Parte 1/programa 1-Bloque 2. Obtenido de https://www.youtube.com/watch?v=M3iMNwBRIAs

Xu, J. (2010). Perception of tourism. Oxford: Elsiever

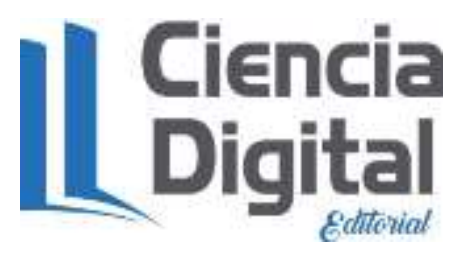




\section{PARA CITAR EL ARTÍCULO INDEXADO.}

Vinueza Chávez, C. A., Aucancela Chimbolema, R., \& Pinta Rodríguez, M. I. (2021). Impacto de la crisis petrolera y la caída en la demanda hotelera en la ciudad del Coca. AlfaPublicaciones, 3(4), 96-116. https://doi.org/10.33262/ap.v3i4.117

\section{Ciencia}

El artículo que se publica es de exclusiva responsabilidad de los autores y no necesariamente reflejan el pensamiento de la Revista Alfa Publicaciones.

El artículo queda en propiedad de la revista y, por tanto, su publicación parcial y/o total en otro medio tiene que ser autorizado por el director de la Revista Alfa Publicaciones.
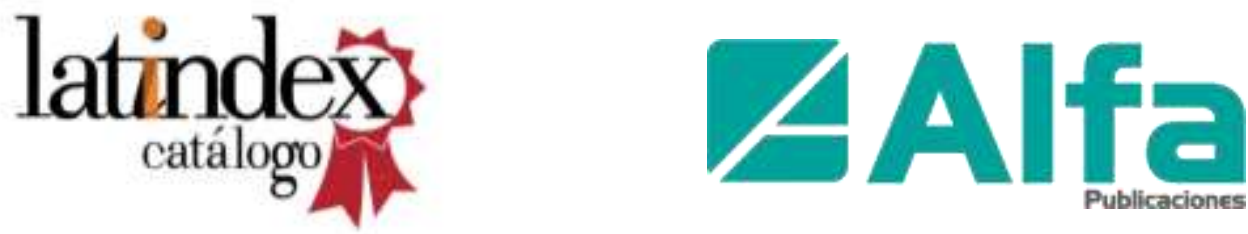